\title{
Improved Quantum Hypercontractivity Inequality for the Qubit Depolarizing Channel
}

\author{
Salman Beigi \\ School of Mathematics, Institute for Research in Fundamental Sciences (IPM) \\ P.O. Box 19395-5746, Tehran, Iran
}

December 9, 2021

\begin{abstract}
The hypercontractivity inequality for the qubit depolarizing channel $\Psi_{t}$ states that $\left\|\Psi_{t}^{\otimes n}(X)\right\|_{p} \leq\|X\|_{q}$ provided that $p \geq q>1$ and $t \geq \ln \sqrt{\frac{p-1}{q-1}}$. In this paper we present an improvement of this inequality. We first prove an improved quantum logarithmic-Sobolev inequality and then use the well-known equivalence of logarithmic-Sobolev inequalities and hypercontractivity inequalities to obtain our main result. As applications of these results, we present an asymptotically tight quantum Faber-Krahn inequality on the hypercube, and a new quantum Schwartz-Zippel lemma.
\end{abstract}

\section{Introduction}

Hypercontractivity inequalities express a bound on the norm of a "noisy" version of a function in terms of the norm of the function itself. When the noise is Markovian and the noise operator belongs to a continuous semigroup, hypercontractivity inequalities are proven using the logSobolev inequalities. Such inequalities bound the entropy of a function in terms its energy, called the Dirichlet form. Hypercontractivity and log-Sobolev inequalities [18, 26, 31, 5, 9] have found several applications, e.g., in concentration of measure inequalities, [10, 29] transportation cost inequalities, [17] estimating the mixing times, [15] analysis of Boolean functions [14] and strong converse bounds in information theory. [1, 19]

Hypercontractivity and log-Sobolev inequalities can be generalized to the (non-commutative) quantum case. [27, 21] In this case, the noise operator is a quantum superoperator and belongs to a quantum Markov semigroup. As in the classical case, quantum hypercontractivity and log-Sobolev inequalities have found several applications (see, e.g., [21, 13, 20, 12, 6, 3, 11, 4]). Nevertheless, due to the non-commutative nature of the quantum theory, proving quantum hypercontractivity and log-Sobolev inequalities is usually more challenging comparing to their classical counterparts.

An important quantum superoperator is the qubit depolarizing channel defined by

$$
\Psi_{t}(X)=e^{-t} X+\left(1-e^{-t}\right) \operatorname{tr}(X) \frac{I}{2}, \quad t \geq 0 .
$$

Here, the superoperators are parametrized in such a way that they satisfy $\Psi_{s} \circ \Psi_{t}=\Psi_{s+t}$ and form a semigroup. The hypercontractivity inequalities for this quantum Markov semigroup, 
first proven in [24, 23], take the form

$$
\left\|\Psi_{t}^{\otimes n}(X)\right\|_{p} \leq\|X\|_{q}, \quad \text { if } \quad e^{2 t} \geq \frac{p-1}{q-1} .
$$

Here, it is assumed that $p, q>1$ and

$$
\|X\|_{p}=\left(\frac{1}{2^{n}} \operatorname{tr}\left(|X|^{p}\right)\right)^{\frac{1}{p}}
$$

where $|X|=\sqrt{X^{\dagger} X}$. This inequality in the classical (commutative) case is a well-known hypercontractivity inequality which takes the same form, but $X$ is restricted to be diagonal in the computational basis (see, e.g., [14]). Moreover, this inequality (without imposing any assumption on $X$ ) is tight even if we restrict $X$ to be diagonal in the computational basis.

The proof of inequality (1) for $n=1$ is a simple consequence of its classical counterpart. Nevertheless, unlike the classical case, its proof for arbitrary $n$ is highly non-trivial. In the commutative case, having (1) for $n=1$, its proof for arbitrary $n$ is immediate using the multiplicativity of the operator norm, or using a certain subadditivity of the entropy function. In the non-commutative case, the latter two properties do not hold in general, so proving (1) for arbitrary $n$ needs new tools. In [24, 23] this inequality is proven using an inequality on the norms of $2 \times 2$ block matrices by King. 22 .

Our main result in this paper is an improvement of (1). Our result is inspired by and is a quantum generalization of the works of Polyanskiy and Samorodnitsky. [30, 28] Again, our result in the case of $n=1$ is easily derived from [30, 28]. Then, to take the induction step we use an entropic inequality of [6], that itself is based on [22].

In the following section after introducing some notations, we formally state our main results in Theorem 2 and Theorem 3. Next, in Section 3 we present some applications. In particular, we present an asymptotically tight quantum Faber-Krahn inequality on the hypercube. Moreover, recalling the Fourier expansion of operators in the Pauli basis and the form of $\Psi_{t}^{\otimes n}$ in that basis, we establish an improved quantum Schwartz-Zippel lemma that puts a bound on the "degree" of an operator in terms of its rank. The proofs of the main theorems, after some auxiliary lemmas in Section 4, come in Sections 6 and 5 . We conclude with some final remarks in Section 7.

\section{Main results}

Throughout the paper, we use $\mathcal{H}=\mathbb{C}^{2}$. We let $\mathcal{B}\left(\mathcal{H}^{\otimes n}\right)$ be the space of linear operators acting $\mathcal{H}^{\otimes n}$, and for $X \in \mathcal{H}^{\otimes n}$ let

$$
\tau(X)=\frac{1}{2^{n}} \operatorname{tr}(X)
$$

be the normalized trace. The Hilbert-Schmidt inner product on $\mathcal{B}\left(\mathcal{H}^{\otimes n}\right)$ is given by

$$
\langle X, Y\rangle:=\tau\left(X^{\dagger} Y\right)
$$

Let $\mathcal{L}: \mathcal{B}(\mathcal{H}) \rightarrow \mathcal{B}(\mathcal{H})$ be the Lindblad generator

$$
\mathcal{L}(X)=X-\tau(X) I .
$$

Then, using $\mathcal{L}^{2}=\mathcal{L}$, the depolarizing channel equals

$$
\Psi_{t}(X)=e^{-t \mathcal{L}}(X)=e^{-t} X+\left(1-e^{-t}\right) \tau(X) I .
$$


The Lindblad generator associated to the $n$-fold tensor product superoperator $\Psi_{t}^{\otimes}$ is equal to

$$
\mathcal{K}_{n}=\sum_{i=1}^{n} \widehat{\mathcal{L}}_{i},
$$

where

$$
\widehat{\mathcal{L}}_{i}:=\mathcal{I}^{\otimes(i-1)} \otimes \mathcal{L} \otimes \mathcal{I}^{\otimes(n-i)}
$$

Here $\mathcal{I}: \mathcal{B}(\mathcal{H}) \rightarrow \mathcal{B}(\mathcal{H})$ is the identity superoperator. Indeed, we have $e^{-t \mathcal{K}_{n}}=\Psi_{t}^{\otimes n}$.

The entropy of a positive semidefinite $X \in \mathcal{B}\left(\mathcal{H}^{\otimes n}\right)$ is given by

$$
\operatorname{Ent}(X)=\tau(X \ln X)-\tau(X) \ln \tau(X) .
$$

The term entropy for this function is used since it is related to the quantum relative entropy; if $X=2^{n} \rho$, where $\rho$ is a density matrix, then $\operatorname{Ent}(X)=\operatorname{tr}(\rho \ln \rho)+\ln 2^{n}=D\left(\rho \| 1 / 2^{n} I\right)$, where $D\left(\rho \| 1 / 2^{n} I\right)$ is the quantum relative entropy between $\rho$ and the maximally mixed state.

The Schatten $p$-norm is defined by

$$
\|X\|_{p}:=\left(\tau\left(|X|^{p}\right)\right)^{\frac{1}{p}}
$$

where as before $|X|=\sqrt{X^{\dagger} X}$. The appearance of the entropy function in the study of hypercontractivity inequalities stems from the fact that the derivative of the norm function is given in terms of entropy.

Lemma 1. Let $X$ be a positive semidefinite matrix. Then the followings hold:

(i) We have

$$
\frac{\mathrm{d}}{\mathrm{d} p}\|X\|_{p}=\frac{1}{p^{2}\|X\|_{p}^{p-1}} \operatorname{Ent}\left(X^{p}\right)
$$

(ii) Let $f(t)=\left\|\Psi_{t}^{\otimes n}(X)\right\|_{p(t)}$. Then we have

$$
f^{\prime}(t)=\frac{1}{\gamma}\left(\frac{p^{\prime}(t)}{p(t)^{2}} \operatorname{Ent}\left(Y^{2}\right)-\left\langle Y^{2 / p(t)}, \mathcal{K}_{n} Y^{2-2 / p(t)}\right\rangle\right),
$$

where $Y=\left(\Psi_{t}^{\otimes n}(X)\right)^{p(t) / 2}$ and $\gamma=\left\|\Psi_{t}^{\otimes n}(X)\right\|_{p(t)}^{p(t)-1}$.

(iii) For any $s>1$ we have

$$
\frac{\operatorname{Ent}\left(X^{s}\right)}{\tau\left(X^{s}\right)} \geq \frac{\ln \|X\|_{s}-\ln \|X\|_{1}}{1-1 / s} .
$$

Proof. Parts (i) and (ii) follow by straightforward computations and are standard (see, e.g., Lemma 12 of [12]). For the third part we use Hölder's inequality for Schatten $p$-norm [7] saying that

$$
\|A B\|_{r} \leq\|A\|_{p}\|B\|_{q},
$$

if $p, q, r>0$ and $1 / r=1 / p+1 / q$. Then for any $x, y \geq 0$ we have

$$
\|X\|_{2 /(x+y)}^{2}=\left\|X^{2}\right\|_{1 /(x+y)} \leq\|X\|_{1 / x} \cdot\|X\|_{1 / y} .
$$




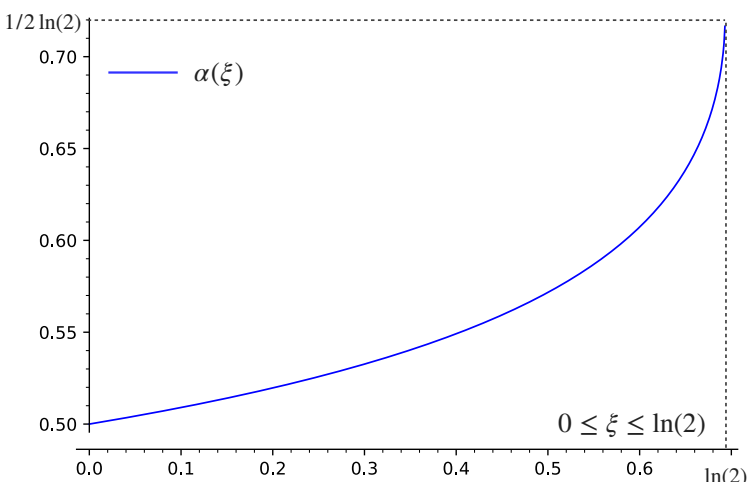

(a)

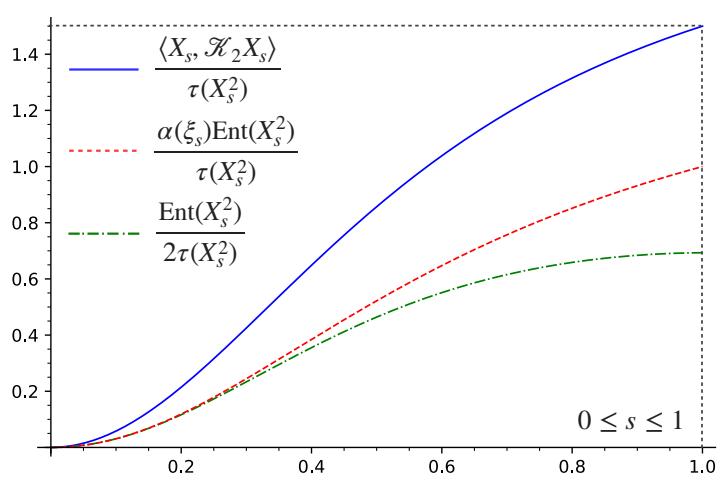

(b)

Figure 1: (a) The plot of function $\alpha(\xi)$ defined in (7). We note that as will be proven in Lemma 8 this function is monotone increasing. (b) For $0 \leq s \leq 1$ let $X_{s}=s|\psi\rangle\langle\psi|+(1-s) \rho \otimes \rho$ where $\rho=I / 2$ and $|\psi\rangle=\frac{1}{\sqrt{2}}(|00\rangle+|11\rangle)$. By Theorem 2, $\alpha\left(\xi_{s}\right) \operatorname{Ent}\left(X_{s}^{2}\right)$ is a lower bound on $\left\langle X_{s}, \mathcal{K}_{2} X_{s}\right\rangle$ that is an improvement over $\frac{1}{2} \operatorname{Ent}\left(X_{s}^{2}\right)$, the standard $\log$-Sobolev inequality. We note that both lower bounds are tight at $s=0$ where $X_{s}$ is a product state, while for larger values of $s$ the improvement provided by our bound becomes apparent. Here, in the plots we divide all the quantities by $\tau\left(X_{s}^{2}\right)$ for the sake of normalization. This is the same as replacing $X_{s}$ with $X_{s} /\left\|X_{s}\right\|_{2}$.

This means that the function

$$
g(x)=\ln \|X\|_{\frac{1}{x}},
$$

is convex. Thus, for all $0<x<1$ we have

$$
g^{\prime}(x) \leq \frac{g(x)-g(1)}{x-1} .
$$

Computing $g^{\prime}(x)$ at $x=1 / s$ using part (i), we obtain the desired inequality.

The binary entropy function for $s \in[0,1]$ is

$$
h(s)=-s \ln s-(1-s) \ln (1-s) .
$$

We note that $h(s)$ is symmetric about $s=1 / 2$ and its restriction to $[0,1 / 2]$ is monotone increasing. Thus, $h^{-1}:[0, \ln 2] \rightarrow[0,1 / 2]$ is well-defined.

We can now state a main result of this paper.

Theorem 2. For every positive semidefinite $X \in \mathcal{B}\left(\mathcal{H}^{\otimes n}\right)$ we have

$$
\alpha(\xi) \cdot \operatorname{Ent}\left(X^{2}\right) \leq\left\langle X, \mathcal{K}_{n} X\right\rangle,
$$

where

$$
\xi=\frac{\operatorname{Ent}\left(X^{2}\right)}{n \tau\left(X^{2}\right)},
$$

and $\alpha:[0, \ln 2] \rightarrow[1 / 2,1 /(2 \ln 2)]$ is given by

$$
\alpha(\xi)=\frac{1}{\xi}\left(\frac{1}{2}-\sqrt{h^{-1}(\ln 2-\xi) \cdot\left(1-h^{-1}(\ln 2-\xi)\right)}\right) .
$$

Remark 1 . In the statement of Theorem 2 we implicitly assume that $\xi$ belongs to the domain of function $\alpha(\cdot)$ which is $[0, \ln 2]$. We will prove this fact in Appendix A 
Remark 2. We note that $\alpha(\xi) \geq 1 / 2$ for all $\xi \in[0, \ln 2]$ (see Figure $1(\mathrm{a})$ ). Thus, inequality (6) is stronger than the log-Sobolev inequality of [21] for the depolarizing channel.

Remark 3. It is not hard to verify that (6) is tight for any $X$ of the form $X=\rho^{\otimes n}$ where $\rho$ is an arbitrary one-qubit positive semidefinite operator.

Let us examine inequality (6) for the choice of $X_{s}=\left|\psi_{s}\right\rangle\left\langle\psi_{s}\right|$ where

$$
\left|\psi_{s}\right\rangle=\sqrt{s}|00\rangle+\sqrt{1-s}|11\rangle \text {. }
$$

Since $X_{s}$ for any $0 \leq s \leq 1$ is a rank-one projection, $\tau\left(X_{s}^{2}\right)=1 / 4$ and $\operatorname{Ent}\left(X_{s}^{2}\right)=\frac{1}{2} \ln (2)$ are independent of $s$, resulting in $\xi_{s}=\operatorname{Ent}\left(X_{s}^{2}\right) / 2 \tau\left(X_{s}^{2}\right)=\ln (2)$. On the other hand, a simple computation verifies that $\left\langle X_{s}, \mathcal{K}_{2} X_{s}\right\rangle=\frac{1}{2}-\frac{1}{4}\left(s^{2}+(1-s)^{2}\right)$. Thus, inequality (6) gives

$$
\alpha\left(\xi_{s}\right) \operatorname{Ent}\left(X_{s}^{2}\right)=\frac{1}{4} \leq \frac{1}{2}-\frac{1}{4}\left(s^{2}+(1-s)^{2}\right),
$$

that is tight at extreme points $s \in\{0,1\}$. This inequality should be compared to the standard $\log$-Sobolev inequality which gives the lower bound of $\frac{1}{2} \operatorname{Ent}\left(X_{s}^{2}\right)=\frac{1}{4} \ln (2)$ on the right hand side that is nowhere tight.

Another interesting example is when $X$ is a mixture of a pure state and a product state. Such an example is illustrated in Figure 1(b).

Using the above improved log-Sobolev inequality, we can establish the following improved quantum hypercontractivity inequality.

Theorem 3. Let $p_{0}>1$ and $X \in \mathcal{B}\left(\mathcal{H}^{\otimes n}\right)$ be such that $\|X\|_{p_{0}} \geq e^{n r_{0}}\|X\|_{1}$ for some $r_{0} \geq 0$. Let $u(t)$ be the solution of the differential equation

$$
\begin{aligned}
& u^{\prime}(t)=\alpha\left(r_{0}\left(1+e^{-u(t)}\right)\right), \\
& u(0)=\ln \left(p_{0}-1\right),
\end{aligned}
$$

where $\alpha(\xi)$ is defined in (7). Then, we have

$$
\left\|\Psi_{t}^{\otimes n}(X)\right\|_{p(t)} \leq\|X\|_{p_{0}},
$$

where $p(t)=1+e^{u(4 t)}$. In particular, for any $t \geq 0$ we have

$$
\left\|\Psi_{t}^{\otimes n}(X)\right\|_{1+\left(p_{0}-1\right) e^{4 \alpha\left(r_{0}\right) t}} \leq\|X\|_{p_{0}} .
$$

Observe that (e.g., by Hölder's inequality) the assumption $\|X\|_{p_{0}} \geq e^{n r_{0}}\|X\|_{1}$ always holds for $r_{0}=0$. In this case, $\alpha\left(r_{0}\right)=1 / 2$, and (10) is equivalent to (1). Thus, choosing the optimal $r_{0} \geq 0$, the above theorem gives improvements of (1).

Remark 4. The differential equation (8) makes sense only if $r_{0}\left(1+e^{-u(t)}\right)$ belongs to the domain of $\alpha(\cdot)$, meaning that $r_{0}\left(1+e^{-u(t)}\right) \leq \ln 2$. To prove this fact observe that by Hölder's inequality (4) we have

$$
\begin{aligned}
\|X\|_{p_{0}} & \leq\left\|X^{1 / p_{0}}\right\|_{p_{0}} \cdot\left\|X^{1-1 / p_{0}}\right\|_{\infty} \\
& =\|X\|_{1}^{1 / p_{0}} \cdot\|X\|_{\infty}^{1-1 / p_{0}} \\
& \leq\|X\|_{1}^{1 / p_{0}} \cdot\left(2^{n}\|X\|_{1}\right)^{1-1 / p_{0}} \\
& =2^{n\left(1-1 / p_{0}\right)}\|X\|_{1} .
\end{aligned}
$$

Then comparing to the assumption $\|X\|_{p_{0}} \geq e^{n r_{0}}\|X\|_{1}$, we find that $r_{0} \leq\left(1-1 / p_{0}\right) \ln 2$. On the other hand, since $\alpha(\cdot)$ is positive, $u(t)$ is monotone increasing and we have $u(t) \geq u(0)=$ $\ln \left(p_{0}-1\right)$. Therefore,

$$
r_{0}\left(1+e^{-u(t)}\right) \leq\left(1-1 / p_{0}\right) \ln 2\left(1+e^{-\ln \left(p_{0}-1\right)}\right)=\ln 2 .
$$




\section{Applications}

In this section, before getting to the proofs of our main results, Theorem 2 and Theorem 3 , we present some applications.

We first note that inequality (6) together with the quantum Stroock-Varopoulos inequality [12, 6] gives other improved log-Sobolev inequalities. In particular, by the quantum StroockVaropoulos inequality we have

$$
\left\langle X, \mathcal{K}_{n} X\right\rangle \leq \frac{1}{4}\left\langle\ln X^{2}, \mathcal{K}_{n} X^{2}\right\rangle
$$

Using this in (6) we obtain

$$
4 \alpha(\xi) \cdot \operatorname{Ent}\left(X^{2}\right) \leq\left\langle\ln X^{2}, \mathcal{K}_{n} X^{2}\right\rangle .
$$

This inequality is an improvement over Theorem 19 of [6] in the qubit case that gives $\operatorname{Ent}\left(X^{2}\right) \leq$ $\left\langle\ln X^{2}, \mathcal{K}_{n} X^{2}\right\rangle$. This is an improvement since $4 \alpha(\xi) \geq 2$. The above inequality is important due to its applications in proving reverse hypercontractivity inequalities [ $[$ ] and bounding the entropy production of quantum channels. [25]

As a second application we present an asymptotically tight quantum version of the FaberKrahn inequality.

Theorem 4. Let $X \in \mathcal{B}\left(\mathcal{H}^{\otimes n}\right)$ be a positive semidefinite operator with rank $R$. Then we have

$$
\frac{1}{2}-\sqrt{h^{-1}\left(\frac{\ln R}{n}\right) \cdot\left(1-h^{-1}\left(\frac{\ln R}{n}\right)\right)} \leq \frac{\left\langle X, \mathcal{K}_{n} X\right\rangle}{n \tau\left(X^{2}\right)} .
$$

We note that this bound is already known to be asymptotically tight in the commutative case in the limit of $n \rightarrow \infty$ when $(\ln R) / n$ is fixed. [16, 30] To this end, let $X$ be a diagonal operator in the computational basis whose support consists of computational basis vectors with bounded hamming weight. See Appendix C of [16] for more details.

Proof. By Theorem 2 we have

$$
\varphi(\xi)=\xi \alpha(\xi) \leq \frac{\left\langle X, \mathcal{K}_{n} X\right\rangle}{n \tau\left(X^{2}\right)}
$$

where $\xi=\operatorname{Ent}\left(X^{2}\right) / n \tau\left(X^{2}\right)$. We note that

$$
\operatorname{Ent}\left(X^{2}\right) \geq \tau\left(X^{2}\right) \ln \frac{\tau\left(X^{2}\right)}{\tau(X)^{2}}
$$

To verify this inequality, we can assume with no loss of generality that $X$ is diagonal and $\tau\left(X^{2}\right)=$ 1. Then, using the concavity of the $\log$ function we have $\tau\left(X^{2} \ln X\right)=-\tau\left(X^{2} \ln X^{-1}\right) \geq$ $-\ln \tau(X)$, that is equivalent to the above inequality. Next, let $P$ be the orthogonal projection on the support of $X$. Then, by the Cauchy-Schwarz inequality we have

$$
\tau(X)=\langle X, P\rangle \leq\|X\|_{2} \cdot\|P\|_{2}=\tau\left(X^{2}\right)^{1 / 2} \cdot\left(\frac{R}{2^{n}}\right)^{1 / 2},
$$

and

$$
\frac{\tau\left(X^{2}\right)}{\tau(X)^{2}} \geq \frac{2^{n}}{R} .
$$


Putting these together we obtain

$$
\xi=\frac{\operatorname{Ent}\left(X^{2}\right)}{n \tau\left(X^{2}\right)} \geq \frac{1}{n} \ln \frac{2^{n}}{R}=\ln 2-\frac{1}{n} \ln R .
$$

Therefore, using the monotonicity of $\varphi(\cdot)$ proven in Lemma 8 below, we have

$$
\varphi\left(\ln 2-\frac{1}{n} \ln R\right) \leq \frac{\left\langle X, \mathcal{K}_{n} X\right\rangle}{n \tau\left(X^{2}\right)}
$$

that is our desired inequality.

For the next application we need to recall the Fourier expansion of qubit operators. 24] Denote the Pauli matrices by

$$
\sigma_{0}=\left(\begin{array}{cc}
1 & 0 \\
0 & 1
\end{array}\right), \quad \sigma_{1}=\left(\begin{array}{cc}
0 & 1 \\
1 & 0
\end{array}\right), \quad \sigma_{2}=\left(\begin{array}{cc}
0 & -i \\
i & 0
\end{array}\right), \quad \sigma_{3}=\left(\begin{array}{cc}
1 & 0 \\
0 & -1
\end{array}\right)
$$

and for $\mathbf{s} \in\{0,1,2,3\}^{n}$ let

$$
\sigma_{\mathbf{s}}:=\otimes_{j=1}^{n} \sigma_{\mathbf{s}_{j}} .
$$

We note that $\left\{\sigma_{\mathbf{s}}: \mathbf{s} \in\{0,1,2,3\}^{n}\right\}$ forms an orthonormal basis for $\mathcal{B}\left(\mathcal{H}^{\otimes n}\right)$ with respect to the Hilbert-Schmidt inner product. Then, any $X \in \mathcal{B}\left(\mathcal{H}^{\otimes n}\right)$ can be written as

$$
X=\sum_{\mathbf{s}} \hat{x}_{\mathbf{s}} \sigma_{\mathbf{s}}
$$

where $\hat{x}_{\mathbf{s}}=\left\langle\sigma_{\mathbf{s}}, X\right\rangle$ are called the Fourier coefficients of $X$. For $\mathbf{s} \in\{0,1,2,3\}^{n}$ we let

$$
|\mathbf{s}|=\left\{j: \mathbf{s}_{j} \neq 0\right\}
$$

and define the degree of $X$ by

$$
\operatorname{deg}(X)=\max \left\{|\mathbf{s}|: \hat{x}_{\mathbf{s}} \neq 0\right\} .
$$

The significance of the Fourier expansion for us is that the superoperator $\Psi_{t}^{\otimes n}(\cdot)$ is diagonal in the Pauli basis. Indeed, for $1 \leq k \leq 3$ we have $\Psi_{t}\left(\sigma_{k}\right)=e^{-t} \sigma_{k}$ and $\Psi_{t}\left(\sigma_{0}\right)=\sigma_{0}$. Therefore,

$$
\Psi_{t}^{\otimes n}\left(\sigma_{\mathbf{s}}\right)=e^{-t|\mathbf{s}|} \sigma_{\mathbf{s}},
$$

and

$$
\Psi_{t}^{\otimes n}(X)=\sum_{\mathbf{s}} e^{-t \mid \mathbf{s}} \hat{x}_{\mathbf{s}} \sigma_{\mathbf{s}}
$$

Now we can state our third application which is a quantum Schwartz-Zippel lemma stating that low-degree operators have high rank.

Theorem 5. Let $0 \neq X \in \mathcal{B}\left(\mathcal{H}^{\otimes n}\right)$ be an operator with rank $e^{n h\left(r_{1}\right)} \leq 2^{n}$ for some $0<r_{1} \leq 1 / 2$, where $h(\cdot)$ is the binary entropy function defined in (5). Then we have

$$
\frac{1}{n} \operatorname{deg}(X) \geq \frac{1}{2}-\sqrt{r_{1}\left(1-r_{1}\right)} .
$$


A quantum Schwartz-Zippel lemma was first proven in Corollary 52 of 24] where it was conjectured that it can be improved. Here, we show that our result is indeed an improvement. Recall that by Corollary 52 of [24] we have

$$
\frac{1}{n} \operatorname{deg}(X) \geq \frac{1}{2}\left(\ln 2-\frac{1}{n} \ln (\operatorname{rank} X)\right)=\frac{1}{2}\left(\ln 2-h\left(r_{1}\right)\right) .
$$

Theorem 5 is an improvement over this bound since we know that $\alpha\left(\ln 2-h\left(r_{1}\right)\right) \geq 1 / 2$ and then

$$
\frac{1}{2}-\sqrt{r_{1}\left(1-r_{1}\right)} \geq \frac{1}{2}\left(\ln 2-h\left(r_{1}\right)\right) .
$$

Proof. For $k \leq n$ define the superoperator $\Pi_{k}: \mathcal{B}\left(\mathcal{H}^{\otimes n}\right) \rightarrow \mathcal{B}\left(\mathcal{H}^{\otimes n}\right)$ by

$$
\Pi_{k}\left(\sigma_{\mathbf{s}}\right)= \begin{cases}\sigma_{\mathbf{s}}, & |\mathbf{s}| \leq k \\ 0, & \text { otherwise }\end{cases}
$$

Then using (11) we have

$$
\Pi_{k} \leq e^{t k} \Psi_{t}^{\otimes n},
$$

meaning that $e^{t k} \Psi_{t}^{\otimes n}-\Pi_{k}$ is a positive semidefinite superoperator. Thus using the fact that $\Pi_{k}$ is a super-projector we have

$$
\begin{aligned}
\left\|\Pi_{k}(X)\right\|_{2}^{2} & =\left\langle X, \Pi_{k}(X)\right\rangle \\
& \leq e^{t k}\left\langle X, \Psi_{t}^{\otimes n}(X)\right\rangle \\
& \leq e^{t k}\|X\|_{q} \cdot\left\|\Psi_{t}^{\otimes n}(X)\right\|_{p},
\end{aligned}
$$

where in the last line we apply Hölder's inequality for $1 \leq q \leq p$ with $1 / p+1 / q=1$.

For $1 \leq q \leq 2$ define $\hat{q}$ by $\frac{1}{\hat{q}}=\frac{1}{q}-\frac{1}{2}$. Also, let $P$ be the projection on the support of $X$. Then, by Hölder's inequality we have

$$
\|X\|_{q}=\|P X\|_{q} \leq\|P\|_{\hat{q}} \cdot\|X\|_{2}=\left(\frac{e^{n h\left(r_{1}\right)}}{2^{n}}\right)^{\frac{1}{\hat{q}}}\|X\|_{2}=e^{-n\left(\ln 2-h\left(r_{1}\right)\right)\left(\frac{1}{q}-\frac{1}{2}\right)}\|X\|_{2},
$$

where in the second equality we use the fact that $P$ is a projection with rank $e^{n h\left(r_{1}\right)}$. Using this for $q=1$, we find that $\|X\|_{2} \geq e^{n r_{0}}\|X\|_{1}$ for

$$
r_{0}=\frac{1}{2}\left(\ln 2-h\left(r_{1}\right)\right)
$$

Thus, we may apply Theorem 3 with $p_{0}=2$. That is,

$$
\left\|\Psi_{t}^{\otimes n}(X)\right\|_{p(t)} \leq\|X\|_{2}, \quad \forall t \geq 0
$$

where $p(t)=1+e^{u(4 t)}$ with $u(0)=0$ and $u^{\prime}(t)=\alpha\left(r_{0}\left(1+e^{-u(t)}\right)\right)$. Then, letting $q(t)=$ $p(t) /(p(t)-1) \leq 2$ be the Hölder conjugate of $p(t)$, we have

$$
\left\|\Pi_{k}(X)\right\|_{2}^{2} \leq e^{t k}\|X\|_{q(t)} \cdot\left\|\Psi_{t}^{\otimes n}(X)\right\|_{p(t)} \leq e^{t k}\|X\|_{q(t)} \cdot\|X\|_{2} .
$$

Next, once again using $(13)$ for $q=q(t) \leq 2$ we obtain

$$
\left\|\Pi_{k}(X)\right\|_{2}^{2} \leq e^{t k} e^{-n\left(\ln 2-h\left(r_{1}\right)\right)\left(\frac{1}{q(t)}-\frac{1}{2}\right)}\|X\|_{2}^{2}=e^{t k} e^{-n\left(\ln 2-h\left(r_{1}\right)\right)\left(\frac{1}{2}-\frac{1}{p(t)}\right)}\|X\|_{2}^{2} .
$$


We note that this inequality holds for any $t \geq 0$. On the other hand, for small values of $t$, using $p^{\prime}(0)=4 u^{\prime}(0) e^{u(0)}=4 \alpha\left(2 r_{0}\right)=4 \alpha\left(\ln 2-h\left(r_{1}\right)\right)$ we have

$$
\frac{1}{p(t)}=\frac{1}{p(0)}-\frac{p^{\prime}(0)}{p(0)^{2}} t+O\left(t^{2}\right)=\frac{1}{2}-\alpha\left(\ln 2-h\left(r_{1}\right)\right) t+O\left(t^{2}\right)
$$

and then

$$
\left\|\Pi_{k}(X)\right\|_{2}^{2} \leq e^{t k} e^{-n\left(\ln 2-h\left(r_{1}\right)\right)\left(\alpha\left(\ln 2-h\left(r_{1}\right)\right) t+O\left(t^{2}\right)\right)}\|X\|_{2}^{2} .
$$

Let $k=\operatorname{deg}(X)$ in which case $\Pi_{k}(X)=X$. Then, since $X \neq 0$, the above inequality says that for all sufficiently small $t>0$ we have

$$
t \operatorname{deg}(X)-n\left(\ln 2-h\left(r_{1}\right)\right)\left(\alpha\left(\ln 2-h\left(r_{1}\right)\right) t+O\left(t^{2}\right)\right) \geq 0 .
$$

Equivalently, this means that

$$
\frac{1}{n} \operatorname{deg}(X) \geq\left(\ln 2-h\left(r_{1}\right)\right) \alpha\left(\ln 2-h\left(r_{1}\right)=\frac{1}{2}-\sqrt{r_{1}\left(1-r_{1}\right)} .\right.
$$

\section{Auxiliary lemmas}

As mentioned above, Theorem 2 and Theorem 3 for $n=1$ are essentially proven in [30, 28, Here, to take the induction step and prove these results for arbitrary $n$, we need to bound the entropy of an operator in $\mathcal{B}\left(\mathcal{H}^{\otimes n}\right)$ in terms of the entropy of certain operators in $\mathcal{B}\left(\mathcal{H}^{\otimes(n-1)}\right)$.

Lemma 6. ([6]) Suppose that $X$ is a positive semidefinite $2 N \times 2 N$ matrix of the block form

$$
X=\left(\begin{array}{cc}
A & C \\
C^{\dagger} & B
\end{array}\right),
$$

where $A, B, C$ are $N \times N$ matrices. Define

$$
M=\left(\begin{array}{cc}
\|A\|_{2} & \|C\|_{2} \\
\left\|C^{\dagger}\right\|_{2} & \|B\|_{2}
\end{array}\right)
$$

Then we have

$$
\operatorname{Ent}\left(X^{2}\right) \leq \operatorname{Ent}\left(M^{2}\right)+\frac{1}{2} \operatorname{Ent}\left(A^{2}\right)+\frac{1}{2} \operatorname{Ent}\left(B^{2}\right)+\operatorname{Ent}\left(|C|^{2}\right) .
$$

This lemma in a more general form is proven in [6]. Here, we briefly explain the proof idea and refer to [6] for a detailed proof.

Proof sketch. For every $p \geq 1$ define

$$
M_{p}=\left(\begin{array}{cc}
\|A\|_{p} & \|C\|_{p} \\
\left\|C^{\dagger}\right\|_{p} & \|B\|_{p}
\end{array}\right) .
$$

Thus $M=M_{2}$. It is shown in 22] that for every $p \geq 2$ we have

$$
\|X\|_{p} \leq\left\|M_{p}\right\|_{p}
$$

That is, letting $g(p):=\left\|M_{p}\right\|_{p}^{p}-\|X\|_{p}^{p}$, we have $g(p) \geq 0$ for all $p \geq 2$. On the other hand, we note that $g(2)=0$. Thus, we must have $g^{\prime}(2) \geq 0$. Computing the derivative of $g(p)$ using Lemma 1 , we find that $g^{\prime}(2) \geq 0$ is equivalent to 16 . 
We need yet another lemma in the proof of Theorem 2 .

Lemma 7. ([6]) For every $C \in \mathcal{B}\left(\mathcal{H}^{\otimes n}\right)$ we have

$$
\left\langle C, \mathcal{K}_{n} C\right\rangle+\left\langle C^{\dagger}, \mathcal{K}_{n} C^{\dagger}\right\rangle \geq\left\langle|C|, \mathcal{K}_{n}|C|\right\rangle+\left\langle\left|C^{\dagger}\right|, \mathcal{K}_{n}\left|C^{\dagger}\right|\right\rangle .
$$

Proof. Observe that

$$
Z_{ \pm}=\left(\begin{array}{ll}
\left|C^{\dagger}\right| & \pm C \\
\pm C^{\dagger} & |C|
\end{array}\right) \geq 0
$$

is positive semidefinite. [8] On the other hand, $\Psi_{t}^{\otimes n}$ is completely positive. Therefore,

$$
\left(\begin{array}{cc}
\Psi_{t}^{\otimes n}\left(\left|C^{\dagger}\right|\right) & \pm \Psi_{t}^{\otimes n}(C) \\
\pm \Psi_{t}^{\otimes n}\left(C^{\dagger}\right) & \Psi_{t}^{\otimes n}(|C|)
\end{array}\right) \geq 0
$$

As a result,

$$
\left\langle\left(\begin{array}{cc}
\left|C^{\dagger}\right| & -C \\
-C^{\dagger} & |C|
\end{array}\right),\left(\begin{array}{cc}
\Psi_{t}^{\otimes n}\left(\left|C^{\dagger}\right|\right) & \Psi_{t}^{\otimes n}(C) \\
\Psi_{t}^{\otimes n}\left(C^{\dagger}\right) & \Psi_{t}^{\otimes n}(|C|)
\end{array}\right)\right\rangle \geq 0 .
$$

This means that

$$
\left\langle|C|, \Psi_{t}^{\otimes n}(|C|)\right\rangle+\left\langle\left|C^{\dagger}\right|, \Psi_{t}^{\otimes n}\left(\left|C^{\dagger}\right|\right)\right\rangle \geq\left\langle C, \Psi_{t}^{\otimes n}(C)\right\rangle+\left\langle C^{\dagger}, \Psi_{t}^{\otimes n}\left(C^{\dagger}\right)\right\rangle .
$$

This inequality holds for all $t \geq 0$, and turns into an equality for $t=0$. Then, the desired inequality follows once we note that

$$
\left.\frac{\mathrm{d}}{\mathrm{d} t} \Psi_{t}^{\otimes n}\right|_{t=0}=-\mathcal{K}_{n}
$$

Our final lemma presents some properties of the function $\alpha(\xi)$ defined in the statement of Theorem 2 ,

Lemma 8. (Theorem 6 of [28] and Lemma 2.1 of [30]) The function $\varphi(\xi)$ defined on the interval $[0, \ln 2]$ by

$$
\varphi(\xi)=\frac{1}{2}-\sqrt{h^{-1}(\ln 2-\xi) \cdot\left(1-h^{-1}(\ln 2-\xi)\right)},
$$

is convex and monotone increasing. Moreover, $\alpha(\xi)=\varphi(\xi) / \xi$ is monotone increasing.

Proof. Let $x \in[0,1 / 2]$ be such that $\xi=\ln 2-h(x)$. Then, we have $\varphi(\xi)=\frac{1}{2}-\sqrt{x(1-x)}$. Taking the derivative of both sides with respect to $x$ we obtain

$$
\ln \left(\frac{1-x}{x}\right) \varphi^{\prime}(\xi)=\frac{1-2 x}{2 \sqrt{x(1-x)}} .
$$

Thus, $\varphi^{\prime}(\xi) \geq 0$ and $\varphi$ is monotone increasing.

Let $y=(1-x) / x \geq 1$. Then, the above equation can be rewritten as

$$
\varphi^{\prime}(\xi)=\frac{1}{2 \ln y} f(y)
$$

where $f(y)=\sqrt{y}-1 / \sqrt{y}$. We note that, $\frac{\mathrm{d}}{\mathrm{d} x} y=-1 / x^{2}$. Therefore, taking the derivative of both sides of the above equation with respect to $x$ we obtain

$$
-\ln (y) \varphi^{\prime \prime}(\xi)=-\frac{1}{2 x^{2}} \frac{\mathrm{d}}{\mathrm{d} y}\left(\frac{1}{\ln y} f(y)\right) .
$$


Hence, to show that $\varphi(\xi)$ is convex, it suffices to show that

$$
\frac{\mathrm{d}}{\mathrm{d} y}\left(\frac{1}{\ln y} f(y)\right) \geq 0
$$

or equivalently

$$
y \ln (y) f^{\prime}(y) \geq f(y) .
$$

Define $g(s)=f\left(e^{2 s}\right)=e^{s}-e^{-s}$ for $s \geq 0$. Then, using $g^{\prime}(s)=2 e^{2 s} f^{\prime}\left(e^{2 s}\right)$ the above inequality is equivalent to

$$
s g^{\prime}(s) \geq g(s) .
$$

This inequality holds since $g(s)=e^{s}-e^{-s}$ is a monotone increasing convex function for $s \geq 0$ and $g(0)=0$.

To verify that $\alpha(\xi)$ is monotone increasing, note that $\alpha^{\prime}(\xi)=\left(\xi \varphi^{\prime}(\xi)-\varphi(\xi)\right) / \xi^{2}$ that is non-negative since $\varphi(\xi)$ is convex, monotone increasing and $\varphi(0)=0$.

\section{Proof of Theorem 2}

As argued in the proof of Lemma 9 in Appendix A, for $n=1$ we may assume that $X^{2}$ is diagonal with eigenvalues $1 \pm r$ for some $r \in[0,1]$. Then $\tau\left(X^{2}\right)=1$ and $\xi=\operatorname{Ent}\left(X^{2}\right)=\ln 2-h((1-r) / 2)$. Therefore,

$$
\alpha(\xi)=\frac{1}{\xi}\left(\frac{1}{2}-\frac{1}{2} \sqrt{1-r^{2}}\right)
$$

and

$$
\alpha(\xi) \cdot \operatorname{Ent}\left(X^{2}\right)=\frac{1}{2}\left(1-\sqrt{1-r^{2}}\right) .
$$

On the other hand, by a simple computation we have

$$
\left\langle X, \mathcal{K}_{1} X\right\rangle=\tau\left(X^{2}\right)-\tau(X)^{2}=\frac{1}{2}\left(1-\sqrt{1-r^{2}}\right) .
$$

Therefore, the desired inequality for $n=1$ holds as an equality.

Now suppose that $n>1$ and that $X \in \mathcal{B}\left(\mathcal{H}^{\otimes n}\right)$ has the block form (14). Let

$$
\varphi(\xi)=\xi \alpha(\xi)=\frac{1}{2}-\sqrt{h^{-1}(\ln 2-\xi) \cdot\left(1-h^{-1}(\ln 2-\xi)\right)} .
$$

We need to show that

$$
n \tau\left(X^{2}\right) \varphi\left(\frac{\operatorname{Ent}\left(X^{2}\right)}{n \tau\left(X^{2}\right)}\right) \leq\left\langle X, \mathcal{K}_{n} X\right\rangle .
$$

By Lemma 6 we have $\operatorname{Ent}\left(X^{2}\right) \leq e_{1}+e_{2}+e_{3}+e_{4}$ where

$$
e_{1}=\operatorname{Ent}\left(M^{2}\right), \quad e_{2}=\frac{1}{2} \operatorname{Ent}\left(A^{2}\right), \quad e_{3}=\frac{1}{2} \operatorname{Ent}\left(B^{2}\right), \quad e_{4}=\operatorname{Ent}\left(|C|^{2}\right) .
$$

On the other hand, a simple computation shows that

$$
\tau\left(X^{2}\right)=\tau\left(M^{2}\right)=\frac{1}{2} \tau\left(A^{2}\right)+\frac{1}{2} \tau\left(B^{2}\right)+\tau\left(|C|^{2}\right),
$$

and

$$
n \tau\left(X^{2}\right)=\tau\left(M^{2}\right)+\frac{n-1}{2} \tau\left(A^{2}\right)+\frac{n-1}{2} \tau\left(B^{2}\right)+(n-1) \tau\left(|C|^{2}\right) .
$$


Therefore, we have

$$
\frac{\operatorname{Ent}\left(X^{2}\right)}{n \tau\left(X^{2}\right)} \leq \frac{e_{1}+e_{2}+e_{3}+e_{4}}{\theta_{1}+\theta_{2}+\theta_{3}+\theta_{4}}
$$

where

$$
\theta_{1}=\tau\left(M^{2}\right), \quad \theta_{2}=\frac{n-1}{2} \tau\left(A^{2}\right), \quad \theta_{3}=\frac{n-1}{2} \tau\left(B^{2}\right), \quad \theta_{4}=(n-1) \tau\left(|C|^{2}\right) .
$$

Using the monotonicity and convexity of $\varphi(\cdot)$ proven in Lemma 8 we have

$$
\begin{aligned}
\varphi\left(\frac{\operatorname{Ent}\left(X^{2}\right)}{n \tau\left(X^{2}\right)}\right) & \leq \varphi\left(\frac{e_{1}+e_{2}+e_{3}+e_{4}}{\theta_{1}+\theta_{2}+\theta_{3}+\theta_{4}}\right) \\
& \leq \sum_{i=1}^{4} \frac{\theta_{i}}{\theta_{1}+\theta_{2}+\theta_{3}+\theta_{4}} \varphi\left(\frac{e_{i}}{\theta_{i}}\right) .
\end{aligned}
$$

This means that

$$
\begin{aligned}
n \tau\left(X^{2}\right) \varphi\left(\frac{\operatorname{Ent}\left(X^{2}\right)}{n \tau\left(X^{2}\right)}\right) \leq & \left(M^{2}\right) \varphi\left(\frac{\operatorname{Ent}\left(M^{2}\right)}{\tau\left(X^{2}\right)}\right)+\frac{n-1}{2} \tau\left(A^{2}\right) \varphi\left(\frac{\operatorname{Ent}\left(A^{2}\right)}{(n-1) \tau\left(A^{2}\right)}\right) \\
& +\frac{n-1}{2} \tau\left(B^{2}\right) \varphi\left(\frac{\operatorname{Ent}\left(B^{2}\right)}{(n-1) \tau\left(B^{2}\right)}\right) \\
& +(n-1) \tau\left(|C|^{2}\right) \varphi\left(\frac{\operatorname{Ent}\left(|C|^{2}\right)}{(n-1) \tau\left(|C|^{2}\right)}\right) .
\end{aligned}
$$

Next, by the induction hypothesis and the fact that $\operatorname{Ent}\left(|C|^{2}\right)=\operatorname{Ent}\left(\left|C^{\dagger}\right|^{2}\right)$ and $\tau\left(|C|^{2}\right)=$ $\tau\left(\left|C^{\dagger}\right|^{2}\right)$, we obtain

$$
\begin{aligned}
n \tau\left(X^{2}\right) \varphi\left(\frac{\operatorname{Ent}\left(X^{2}\right)}{n \tau\left(X^{2}\right)}\right) \leq\langle & M, \mathcal{L} M\rangle+\frac{1}{2}\left\langle A, \mathcal{K}_{n-1} A\right\rangle+\frac{1}{2}\left\langle B, \mathcal{K}_{n-1} B\right\rangle \\
& +\frac{1}{2}\left\langle|C|, \mathcal{K}_{n-1}|C|\right\rangle+\frac{1}{2}\left\langle\left|C^{\dagger}\right|, \mathcal{K}_{n-1}\left|C^{\dagger}\right|\right\rangle .
\end{aligned}
$$

We now note that

$$
\left\langle X, \mathcal{K}_{n} X\right\rangle=\left\langle X, \mathcal{L} \otimes \mathcal{I}^{\otimes(n-1)}(X)\right\rangle+\left\langle\left(\begin{array}{cc}
A & C \\
C^{\dagger} & B
\end{array}\right),\left(\begin{array}{cc}
\mathcal{K}_{n-1}(A) & \mathcal{K}_{n-1}(C) \\
\mathcal{K}_{n-1}\left(C^{\dagger}\right) & \mathcal{K}_{n-1}(B)
\end{array}\right)\right\rangle .
$$

We compute each term in the above sum separately:

$$
\begin{aligned}
\left\langle X, \mathcal{L} \otimes \mathcal{I}^{\otimes(n-1)}(X)\right\rangle & =\left\langle\left(\begin{array}{cc}
A & C \\
C^{\dagger} & B
\end{array}\right),\left(\begin{array}{cc}
\frac{A-B}{2} & C \\
C^{\dagger} & \frac{B-A}{2}
\end{array}\right)\right\rangle \\
& =\frac{1}{2}\left(\frac{1}{2} \tau\left(A^{2}\right)+\frac{1}{2} \tau\left(B^{2}\right)-\tau(A B)+2 \tau\left(C^{\dagger} C\right)\right) \\
& \geq \frac{1}{2}\left(\frac{1}{2}\|A\|_{2}^{2}+\frac{1}{2}\|B\|_{2}^{2}-\|A\|_{2} \cdot\|B\|_{2}+2\|C\|_{2}^{2}\right) \\
& =\langle M, \mathcal{L} M\rangle .
\end{aligned}
$$


For the second term we have

$$
\begin{aligned}
\left\langle\left(\begin{array}{cc}
A & C \\
C^{\dagger} & B
\end{array}\right),\left(\begin{array}{cc}
\mathcal{K}_{n-1}(A) & \mathcal{K}_{n-1}(C) \\
\mathcal{K}_{n-1}\left(C^{\dagger}\right) & \mathcal{K}_{n-1}(B)
\end{array}\right)\right\rangle= & \frac{1}{2}\left\langle A, \mathcal{K}_{n-1} A\right\rangle+\frac{1}{2}\left\langle B, \mathcal{K}_{n-1} B\right\rangle \\
& +\frac{1}{2}\left\langle C, \mathcal{K}_{n-1} C\right\rangle+\frac{1}{2}\left\langle C^{\dagger}, \mathcal{K}_{n-1} C^{\dagger}\right\rangle \\
\geq & \frac{1}{2}\left\langle A, \mathcal{K}_{n-1} A\right\rangle+\frac{1}{2}\left\langle B, \mathcal{K}_{n-1} B\right\rangle \\
& +\frac{1}{2}\left\langle|C|, \mathcal{K}_{n-1}|C|\right\rangle+\frac{1}{2}\left\langle\left|C^{\dagger}\right|, \mathcal{K}_{n-1}\left|C^{\dagger}\right|\right\rangle
\end{aligned}
$$

where the inequality follows from Lemma 7. Putting these in (17) the desired inequality follows.

\section{Proof of Theorem 3}

Let us first assume that $X$ is positive semidefinite. Later we will show how to generalize the argument for arbitrary $X$. Define

$$
f(t)=\left\|\Psi_{t}^{\otimes n}(X)\right\|_{p(t)}-\|X\|_{p_{0}} .
$$

We need to show that $f(t) \leq 0$ for all $t \geq 0$. We note that $f(0)=0$. Suppose that for some $t_{1}>0$ we have $f\left(t_{1}\right)>0$. Let

$$
s_{0}=\sup \left\{s \mid 0 \leq s<t_{1}, f(s)=0\right\} .
$$

By the continuity of $f(t)$ we have $f\left(s_{0}\right)=0$. Moreover, $f$ is positive in the interval $\left(s_{0}, t_{1}\right)$. Then, by the mean value theorem there exits $t_{0} \in\left(s_{0}, t_{1}\right)$ such that

$$
f\left(t_{0}\right) \geq 0,
$$

and

$$
f^{\prime}\left(t_{0}\right)=f\left(t_{1}\right) /\left(t_{1}-s_{0}\right)>0
$$

By Lemma 1 we have

$$
\left\|\Psi_{t_{0}}^{\otimes n}\right\|_{p\left(t_{0}\right)}^{p\left(t_{0}\right)-1} \cdot f^{\prime}\left(t_{0}\right)=\frac{p^{\prime}\left(t_{0}\right)}{p\left(t_{0}\right)^{2}} \operatorname{Ent}\left(Y^{2}\right)-\left\langle Y^{2 / p\left(t_{0}\right)}, \mathcal{K}_{n} Y^{2-2 / p\left(t_{0}\right)}\right\rangle,
$$

where $Y=\left(\Psi_{t_{0}}^{\otimes n}(X)\right)^{p\left(t_{0}\right) / 2}$. Thus $f^{\prime}\left(t_{0}\right)>0$ implies

$$
\frac{p^{\prime}\left(t_{0}\right)}{p\left(t_{0}\right)^{2}} \operatorname{Ent}\left(Y^{2}\right)>\left\langle Y^{2 / p\left(t_{0}\right)}, \mathcal{K}_{n} Y^{2-2 / p\left(t_{0}\right)}\right\rangle .
$$

Next, using the quantum Stroock-Varopoulos inequality [12, 6] stating that

$$
\left\langle Y^{2 / p\left(t_{0}\right)}, \mathcal{K}_{n} Y^{2-2 / p\left(t_{0}\right)}\right\rangle \geq \frac{4\left(p\left(t_{0}\right)-1\right)}{p\left(t_{0}\right)^{2}}\left\langle Y, \mathcal{K}_{n} Y\right\rangle
$$

we conclude that

$$
\frac{p^{\prime}\left(t_{0}\right)}{4\left(p\left(t_{0}\right)-1\right)} \operatorname{Ent}\left(Y^{2}\right)>\left\langle Y, \mathcal{K}_{n} Y\right\rangle
$$

On the other hand, Theorem 2 gives

$$
\alpha(\xi) \cdot \operatorname{Ent}\left(Y^{2}\right) \leq\left\langle Y, \mathcal{K}_{n} Y\right\rangle,
$$


where $\xi=\operatorname{Ent}\left(Y^{2}\right) /\left(n \tau\left(Y^{2}\right)\right)$. Then comparing the above two inequalities, we find that

$$
\alpha(\xi)<\frac{p^{\prime}\left(t_{0}\right)}{4\left(p\left(t_{0}\right)-1\right)} .
$$

Next, using $p^{\prime}\left(t_{0}\right)=4 u^{\prime}\left(t_{0}\right) e^{u\left(4 t_{0}\right)}$ and the given differential equation for $u(t)$ we have

$$
\frac{p^{\prime}\left(t_{0}\right)}{4\left(p\left(t_{0}\right)-1\right)}=\alpha\left(\frac{r_{0} p\left(t_{0}\right)}{p\left(t_{0}\right)-1}\right) \text {. }
$$

Comparing the above two inequalities and the monotonicity of $\alpha(\cdot)$ stated in Lemma 8, we obtain

$$
\xi<\frac{r_{0} p\left(t_{0}\right)}{p\left(t_{0}\right)-1}
$$

Now, part (iii) of Lemma 1 yields

$$
\frac{\operatorname{Ent}\left(Y^{2}\right)}{\tau\left(Y^{2}\right)}=\frac{\operatorname{Ent}\left(\Psi_{t_{0}}^{\otimes n}(X)^{p\left(t_{0}\right)}\right)}{\tau\left(\Psi_{t_{0}}^{\otimes n}(X)^{p\left(t_{0}\right)}\right)} \geq \frac{\ln \left\|\Psi_{t_{0}}^{\otimes n}(X)\right\|_{p\left(t_{0}\right)}-\ln \left\|\Psi_{t_{0}}^{\otimes n}(X)\right\|_{1}}{1-1 / p\left(t_{0}\right)}
$$

On the other hand, by (18) we have $\left\|\Psi_{t_{0}}^{\otimes n}(X)\right\|_{p\left(t_{0}\right)} \geq\|X\|_{p_{0}}$. Moreover, $X$ and $\Psi_{t_{0}}^{\otimes n}(X)$ are positive semidefinite. Hence,

$$
\left\|\Psi_{t_{0}}^{\otimes n}(X)\right\|_{1}=\tau\left(\Psi_{t_{0}}^{\otimes n}(X)\right)=\tau(X)=\|X\|_{1} .
$$

Putting these together we conclude that

$$
\frac{\operatorname{Ent}\left(Y^{2}\right)}{\tau\left(Y^{2}\right)} \geq \frac{\ln \|X\|_{p_{0}}-\ln \|X\|_{1}}{1-1 / p\left(t_{0}\right)} \geq \frac{n r_{0}}{1-1 / p\left(t_{0}\right)},
$$

which means

$$
\xi>\frac{r_{0} p\left(t_{0}\right)}{p\left(t_{0}\right)-1}
$$

This is in contradiction with 19 .

Now assume that $X$ is arbitrary. Let $Z=|X|=\sqrt{X^{\dagger} X}$ and $Z^{\prime}=\left|X^{\dagger}\right|=\sqrt{X X^{\dagger}}$. Then since $\Psi_{t}(\cdot)$ is completely positive, we have $\left\|\Psi_{t}(X)\right\|_{q}^{2} \leq\left\|\Psi_{t}(Z)\right\|_{q} \cdot\left\|\Psi_{t}\left(Z^{\prime}\right)\right\|_{q}$ for any $q \geq 1$. This fact in the special case of hermitian $X$ was first showed in [2], and its general form can be inferred from the proof of Lemma 2 of [32. Here we present another proof. As already shown in the proof of Lemma 7 we have

$$
\left(\begin{array}{cc}
\Psi_{t}^{\otimes n}\left(Z^{\prime}\right) & \Psi_{t}^{\otimes n}(X) \\
\Psi_{t}^{\otimes n}\left(X^{\dagger}\right) & \Psi_{t}^{\otimes n}(Z)
\end{array}\right)=\left(\begin{array}{cc}
\Psi_{t}^{\otimes n}\left(\left|X^{\dagger}\right|\right) & \Psi_{t}^{\otimes n}(X) \\
\Psi_{t}^{\otimes n}\left(X^{\dagger}\right) & \Psi_{t}^{\otimes n}(|X|)
\end{array}\right) \geq 0
$$

This means that there is a contraction $K$ such that (see Proposition 1.3.2 of [8])

$$
\Psi_{t}^{\otimes n}(X)=\sqrt{\Psi_{t}^{\otimes n}\left(Z^{\prime}\right)} K \sqrt{\Psi_{t}^{\otimes n}(Z)} .
$$

Therefore, by Hölder's inequality

$$
\begin{aligned}
\left\|\Psi_{t}^{\otimes n}(X)\right\|_{q} & =\left\|\sqrt{\Psi_{t}^{\otimes n}\left(Z^{\prime}\right)} K \sqrt{\Psi_{t}^{\otimes n}(Z)}\right\|_{q} \leq\left\|\sqrt{\Psi_{t}^{\otimes n}\left(Z^{\prime}\right)}\right\|_{2 q} \cdot\|K\|_{\infty} \cdot\left\|\sqrt{\Psi_{t}^{\otimes n}(Z)}\right\|_{2 q} \\
& \leq\left\|\sqrt{\Psi_{t}^{\otimes n}\left(Z^{\prime}\right)}\right\|_{2 q} \cdot\left\|\sqrt{\Psi_{t}^{\otimes n}(Z)}\right\|_{2 q} \\
& =\left\|\Psi_{t}^{\otimes n}\left(Z^{\prime}\right)\right\|_{q}^{1 / 2} \cdot\left\|\Psi_{t}^{\otimes n}(Z)\right\|_{q}^{1 / 2}
\end{aligned}
$$


Next, using the fact that $\|Z\|_{q}=\|X\|_{q}=\left\|X^{\dagger}\right\|_{q}=\left\|Z^{\prime}\right\|_{q}$ we find that by assumption $\|Z\|_{p_{0}}=$ $\left\|Z^{\prime}\right\|_{p_{0}}=\|X\|_{p_{0}} \geq e^{n r_{0}}\|X\|_{1}=e^{n r_{0}}\|Z\|_{1}=e^{n r_{0}}\left\|Z^{\prime}\right\|_{1}$. Then, as we showed that the theorem holds in the case of positive semidefinite operators $Z, Z^{\prime}$, we have

$$
\begin{aligned}
\left\|\Psi_{t}^{\otimes n}(X)\right\|_{p(t)} & \leq\left\|\Psi_{t}^{\otimes n}(Z)\right\|_{p(t)}^{1 / 2} \cdot\left\|\Psi_{t}^{\otimes n}\left(Z^{\prime}\right)\right\|_{p(t)}^{1 / 2} \\
& \leq\|Z\|_{p_{0}}^{1 / 2} \cdot\left\|Z^{\prime}\right\|_{p_{0}}^{1 / 2} \\
& =\|X\|_{p_{0}} .
\end{aligned}
$$

To prove $(10)$, note that by Lemma 8 the function $\alpha(\xi)$ is monotone non-decreasing. Therefore, $u^{\prime}(t) \geq \alpha\left(r_{0}\right)$ for all $t$, and $u(t) \geq \alpha\left(r_{0}\right)+\ln \left(p_{0}-1\right)$. Using this in (9), and using the monotonicity of $q \mapsto\|X\|_{q}$, which can be proven using Hölder's inequality, we obtain (10).

\section{Final remarks}

One may wonder whether the above results can be extended to the generalized depolarizing semigroup with the Lindblad generator $\mathcal{L}^{\prime}(X)=X-\operatorname{tr}(\eta X) I$, where $\eta$ is an arbitrary full-rank qubit state (see, e.g., [6]). We note that both Lemma 6 and Lemma 7, as our main tools in proving our results, already hold for arbitrary such $\eta$ 's. Thus, it seems that the induction step in the proof of Theorem 2 can be reproduced for arbitrary $\eta$. Nevertheless, a main issue in such a generalization is the definitions of functions $\alpha(\cdot)$ and $\varphi(\cdot)$ when $\eta$ is not the maximally mixed state. We note that, the functions $\alpha(\cdot)$ and $\varphi(\cdot)$ in the case of $\eta=I / 2$ are defined in such a way that for $n=1$ we get equality in (6). Moreover, we saw that luckily $\varphi(\cdot)$ defined in this way is convex, and this convexity is used to execute the induction step. We may take the same path and define $\varphi(\cdot)$ for arbitrary $\eta$ by assuming equality in (6) for $n=1$. However, even restricting to the completely classical setting (diagonal operators), such a function $\varphi(\cdot)$ would not be convex in general. An approach to overcome this obstacle is to simply replace such a $\varphi(\cdot)$ with its lower convex envelop, i.e., the largest convex function that is point-wise smaller. We leave exploring such a generalization for future works.

The improved log-Sobolev inequality of Theorem 2 is indeed a log-Sobolev inequality for the parameter $p=2$ (see, e.g., [6] for more details). It would be interesting to extend this result to other values of $p$, as done in [28] for the classical case. In particular, if we could optimally generalize Theorem 2 to the case of $p=1$ (an improved quantum modified logSobolev inequality), we would be able to prove a quantum version of Mrs. Gerber's Lemma through the approach of [28]. As Mrs. Gerber's Lemma has several applications in information theory, a quantum version would also be desirable and would find applications. We note that as done in Section 3 , the quantum Stroock-Varopoulos inequality and Theorem 2 provide us with an improved modified log-Sobolev inequality, but not an optimal one as in the case of $p=2$ (see Remark 3). To obtain such an optimal inequality we should take a direct approach. To this end, a main difficulty is to replace the inequality of Lemma 6 to something relevant to the case of $p=1$.

\section{A Bounding the entropy in terms of 2-norm}

Lemma 9. For every positive semidefinite $X \in \mathcal{B}\left(\mathcal{H}^{\otimes n}\right)$ we have

$$
\xi=\frac{\operatorname{Ent}\left(X^{2}\right)}{n \tau\left(X^{2}\right)} \in[0, \ln 2] .
$$


Proof. We need to show that

$$
\operatorname{Ent}\left(X^{2}\right) \leq n \ln (2) \tau\left(X^{2}\right) .
$$

We prove this by induction on $n$. First, let $n=1$. In this case, both $\operatorname{Ent}\left(X^{2}\right)$ and $\tau\left(X^{2}\right)$ depend only on the eigenvalues of $X^{2}$. Next, by a scaling argument we can assume with no loss of generality that the eigenvalues of $X^{2}$ are $1 \pm r$ for some $r \in[0,1]$. Then $\tau\left(X^{2}\right)=1$ and we have

$$
\begin{aligned}
\operatorname{Ent}\left(X^{2}\right) & =\frac{1}{2}((1+r) \ln (1+r)+(1-r) \ln (1-r)) \\
& \leq \frac{1}{2}(1+r) \ln (1+r) \\
& \leq \ln 2 .
\end{aligned}
$$

Now suppose that $n>1$, and that $X$ is of the block form 14 with $A, B, C \in \mathcal{B}\left(\mathcal{H}^{\otimes(n-1)}\right)$. By Lemma 6 we have

$$
\operatorname{Ent}\left(X^{2}\right) \leq \operatorname{Ent}\left(M^{2}\right)+\frac{1}{2} \operatorname{Ent}\left(A^{2}\right)+\frac{1}{2} \operatorname{Ent}\left(B^{2}\right)+\operatorname{Ent}\left(|C|^{2}\right) .
$$

On the other hand, a simple computation shows that

$$
\tau\left(X^{2}\right)=\tau\left(M^{2}\right)=\frac{1}{2} \tau\left(A^{2}\right)+\frac{1}{2} \tau\left(B^{2}\right)+\tau\left(|C|^{2}\right),
$$

and

$$
n \tau\left(X^{2}\right)=\tau\left(M^{2}\right)+\frac{n-1}{2} \tau\left(A^{2}\right)+\frac{n-1}{2} \tau\left(B^{2}\right)+(n-1) \tau\left(|C|^{2}\right) .
$$

Using the induction hypothesis for $M, A, B,|C|$, and the above equations, the desired inequality follows.

\section{References}

[1] R. Ahlswede and P. Gacs, "Spreading of sets in product spaces and hypercontraction of the Markov operator," Ann. Probab. 4(6), 925-939 (1976).

[2] G. G. Amosov and A. S. Holevo, "On the multiplicativity hypothesis for quantum communication channels," Theory Probab. Appl. 47(1), 123-127 (2020).

[3] I. Bardet, A. Capel, A. Lucia, D. Pérez-García, and C. Rouzé, "On the modified logarithmic Sobolev inequality for the heat-bath dynamics for 1D systems," J. Math. Phys. 62(6), 061901 (2021).

[4] I. Bardet, M. Junge, N. LaRacuente, C. Rouzé, and D. S. França, "Group transference techniques for the estimation of the decoherence times and capacities of quantum Markov semigroups," IEEE Trans. Inf. Theory 67(5), 2878-2909, (2021).

[5] W. Beckner, "Inequalities in Fourier analysis," Ann. of Math. 102(1), 159-182 (1975).

[6] S. Beigi, N. Datta, C. Rouzé, "Quantum reverse hypercontractivity: its tensorization and application to strong converses," Commun. Math. Phys. 376(2), 753-94 (2020).

[7] R. Bhatia, Matrix Analysis (Graduate Texts in Mathematics 169, Springer, 1997).

[8] R. Bhatia, Positive definite matrices (Princeton university press, 2009).

[9] A. Bonami, "Étude des coefficients de Fourier des fonctions de $L_{p}(G)$," Ann. Inst. Fourier 20(2), 335-402 (1970).

[10] S. Boucheron, G. Lugosi, and P. Massart, Concentration Inequalities: A Nonasymptotic Theory of Independence (Oxford University Press, 2013). 
[11] H.C. Cheng, N. Datta, and C. Rouzé, "Strong converse for classical-quantum degraded broadcast channels," arXiv preprint arXiv:1905.00874 (2019).

[12] T. Cubitt, M. Kastoryano, A. Montanaro, K. Temme, "Quantum reverse hypercontractivity," J. Math. Phys. 56(10), 102204 (2015).

[13] P. Delgosha and S. Beigi, "Impossibility of Local State Transformation via Hypercontractivity," Commun. Math. Phys. 332, 449-476 (2014).

[14] R. de Wolf, "A Brief Introduction to Fourier Analysis on the Boolean Cube," Theory of Computing, Graduate Surveys 1, 1-20 (2008).

[15] P. Diaconis, L. Saloff-Coste, "Logarithmic Sobolev inequalities for finite Markov chains," Ann. Appl. Probab. 6(3) 695-750 (1996).

[16] J. Friedman and J. P. Tillich, "Generalized Alon-Boppana Theorems and Error-Correcting Codes," SIAM J. Discrete Math. 19(3), 700-718 (2005).

[17] N. Gozlan and C. Léonard, "Transport inequalities, a survey," Markov Process. Relat. Fields, 16, 635-736 (2010).

[18] L. Gross, "Logarithmic Sobolev inequalities," Am. J. Math. 97(4), 1061-1083 (1975).

[19] S. Kamath, and V. Anantharam, "Non-interactive simulation of joint distributions: The Hirschfeld-GebeleinRényi maximal correlation and the hypercontractivity ribbon," In 2012 50th Annu. Allert. Conf. Commun. Control Comput. (Allerton), 1057-1064 (2012).

[20] M. J. Kastoryano, F. Pastawski and K. Temme, "Hypercontractivity of quasi-free quantum semigroups," J. Phys. A: Math. Theor. 47(40), 405303 (2014).

[21] M. Kastoryano, and K. Temme, "Quantum logarithmic Sobolev inequalities and rapid mixing," J. Math. Phys. 54(5), 052202 (2013).

[22] C. King, "Inequalities for Trace Norms of $2 \times 2$ Block Matrices," Commun. Math. Phys. 242(3) 531-545 (2003).

[23] C. King, "Hypercontractivity for semigroups of unital qubit channels," Commun. Math. Phys. 328(1), 285301 (2014).

[24] A. Montanaro and T. Osborne, "Quantum Boolean Functions," Chicago J. Theor. Comput. Sci. 2010(1), (2010).

[25] A. Müller-Hermes, D. Stilck Franca and M. M. Wolf, "Entropy production of doubly stochastic quantum channels," J. Math. Phys. 57(2), 022203 (2016)

[26] E. Nelson, "A quartic interaction in two dimensions," In Mathematical Theory of Elementary Particles, MIT Press, 69-73 (1966).

[27] R. Olkiewicz and B. Zegarlinski, "Hypercontractivity in Noncommutative $L_{p}$ Spaces," J. Funct. Anal. 161(1), 246-285 (1999).

[28] Y. Polyanskiy, A Samorodnitsky, "Improved log-Sobolev inequalities, hypercontractivity and uncertainty principle on the hypercube," J. Funct. Anal. 277(11), 108280 (2019).

[29] M. Raginsky and I. Sason, "Concentration of measure inequalities in information theory, communications, and coding," Found. Trends@ Commun. Inf. Theory 10(1-2), 1-246 (2013).

[30] S. Samorodnitsky, "A modified logarithmic Sobolev inequality for the Hamming cube and some applications," arXiv preprint arXiv:0807.1679 (2008).

[31] B. Simon and R. Hoegh-Krohn, "Hypercontractive semigroups and two dimensional self-coupled Bose fields," J. Funct. Anal. 9(2), 121-180 (1972).

[32] J. Watrous, "Notes on super-operator norms induced by Schatten norms," Quantum Inf. Comput. 5(1), 58-68 (2005). 